Supporting information for

\title{
Redissolution of Flower-shaped Graphene Oxide Powder with High Density
}

\author{
Chen Chen ${ }^{\dagger}$, Zhen Xu $u^{\dagger}, Y_{\text {Han }}^{\star}$,Haiyan Sun and Chao Gao $*^{\dagger}$ \\ ${ }^{\dagger}$ MOE Key Laboratory of Macromolecular Synthesis and Functionalization, \\ Department of Polymer Science and Engineering, Zhejiang University, Hangzhou \\ 310027, P. R. China. \\ ${ }^{\ddagger}$ ZJU-C6G6 Joint Graphene Centre, 38 Zheda Road, Hangzhou, P. R. China.
}

*To whom correspondence should be addressed. E-mail: chaogao@zju.edu.cn 
The SI file includes:

Figure S1.SEM images and grain size distribution of fdGO.

Figure S2. TEM and AFM images of dissolved fdGO.

Figure S3. XPS and Raman spectra of pristine GO and fdGO.

Figure S4.Digital pictures of fGO prepared under $200^{\circ} \mathrm{C}$ and its aqueous dispersion.

Figure S5. Digital pictures of compressed GOA and its aqueous dispersion.

Figure S6. High-solution optical images of fdGO during dissolution.

Figure S7.UV-Vis spectra of aqueous dispersion of GOA.

Figure S8.SEM images of fdGO after being dispersed in DMF, ethanol and acetone.

Figure S9. Digital pictures of GO dispersed in 14 different solvents.

Figure S10.Digital picture of a Acorn $\mathrm{Area}^{\mathrm{TM}}$ and its schematic diagram. 

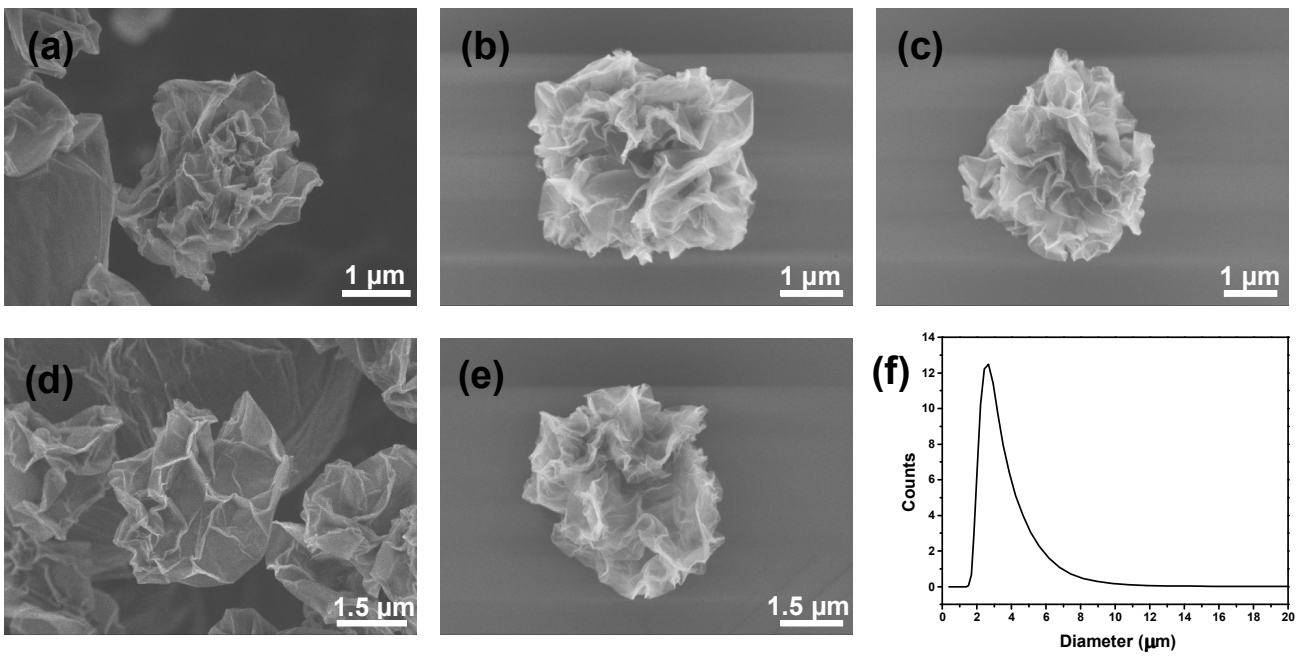

Figure S1. (a-e) SEM images of individual fGO. (f) Grain size distribution of fGO 
(a)
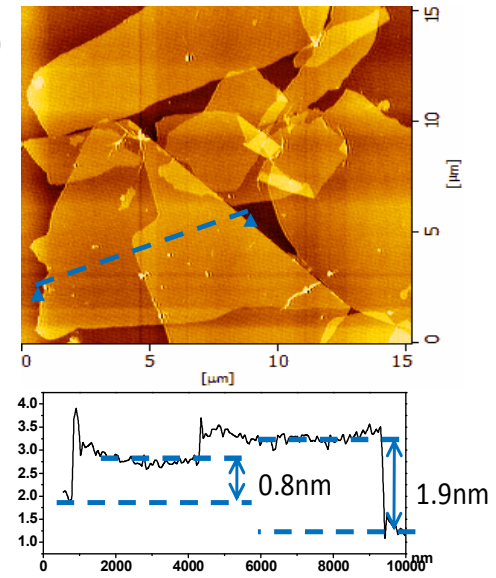

(c)

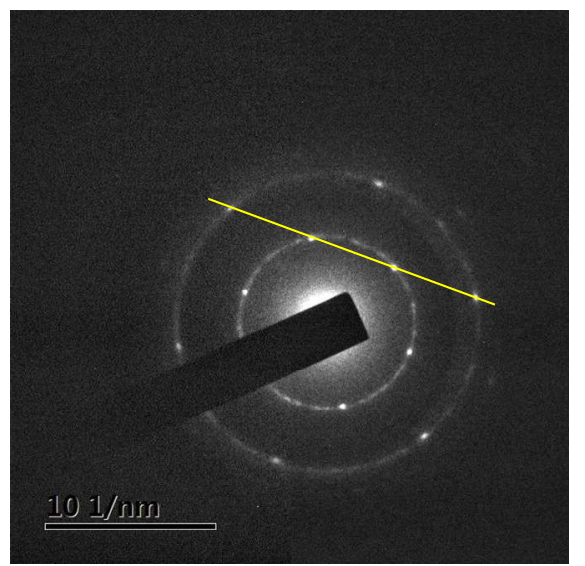

(b)

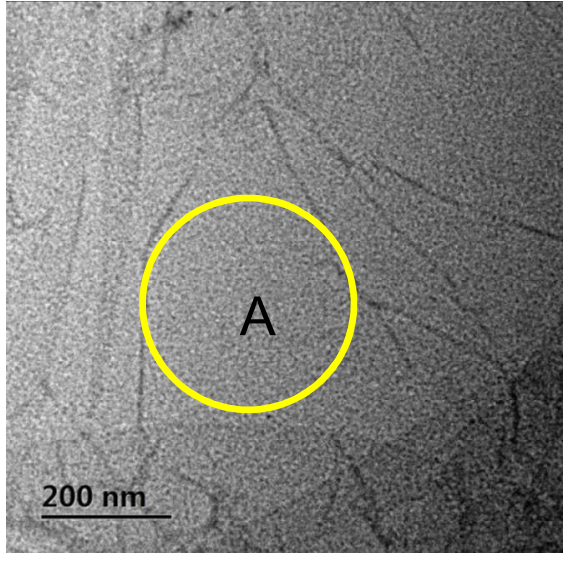

(d)

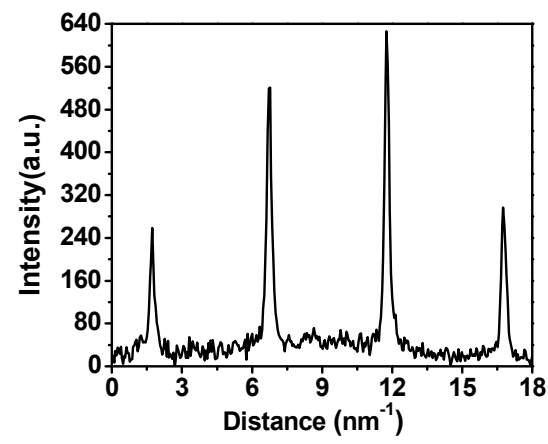

Figure S2. (a) Tapping mode AFM image and height profile of fGO after agitation for 24h. (b,c) TEM image of fGO after agitation for $24 \mathrm{~h}$ (b), and its selected area electron diffraction (SAED) diffraction patterns (c). (d) Intensity profile through the diffraction spots labeled in (c). 

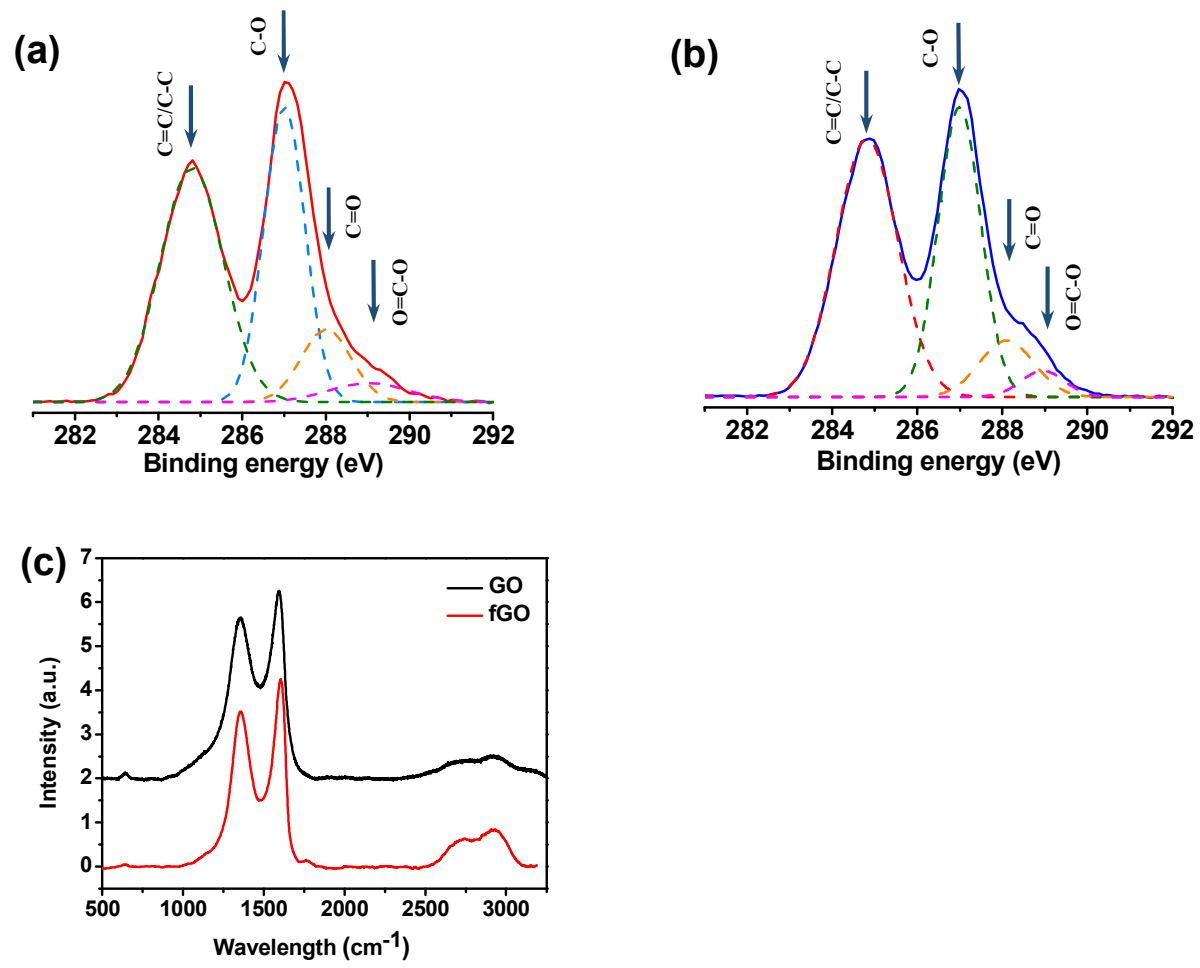

Figure S3. (a,b) C1s XPS spectra of pristine GO (a) and fdGO (b). (c) Raman spectra of GO and fGO. Both GO and fGO show two characteristic D and G bands at 1365 and $1615 \mathrm{~cm}^{-1}$. The intensity ratio $\left(\mathrm{I}_{\mathrm{D}} / \mathrm{I}_{\mathrm{G}}\right)$ of fGO $(0.831)$ is close to that of $\mathrm{GO}$ (0.848). 


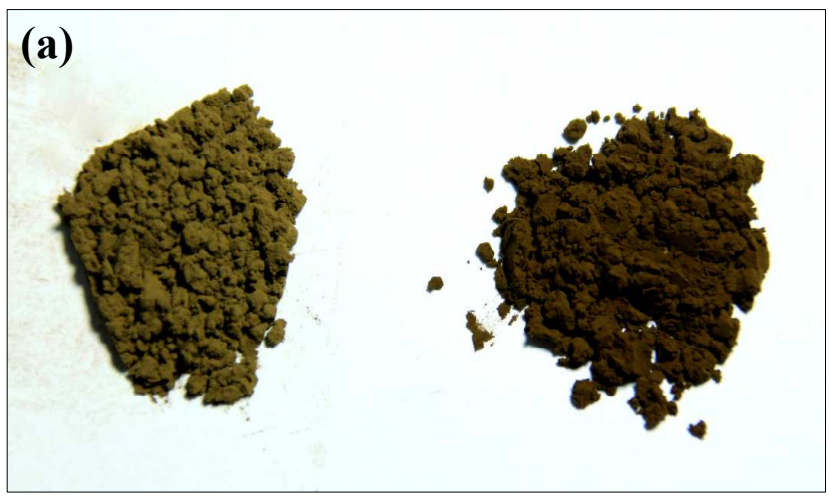

(b)

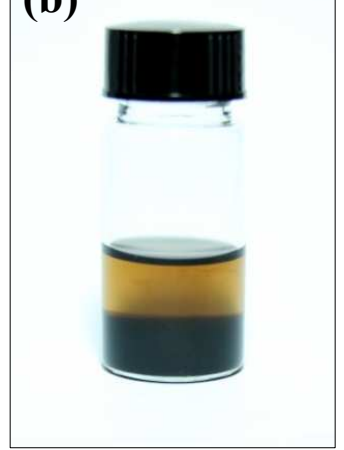

Figure S4. (a) Comparison of fGO (left) and fGO prepared under $200{ }^{\circ} \mathrm{C}$ (named as fGO-200, right). (b) Digital picture of fGO-200 after stirred in water for $24 \mathrm{~h}$, followed by settling for $24 \mathrm{~h}$. 


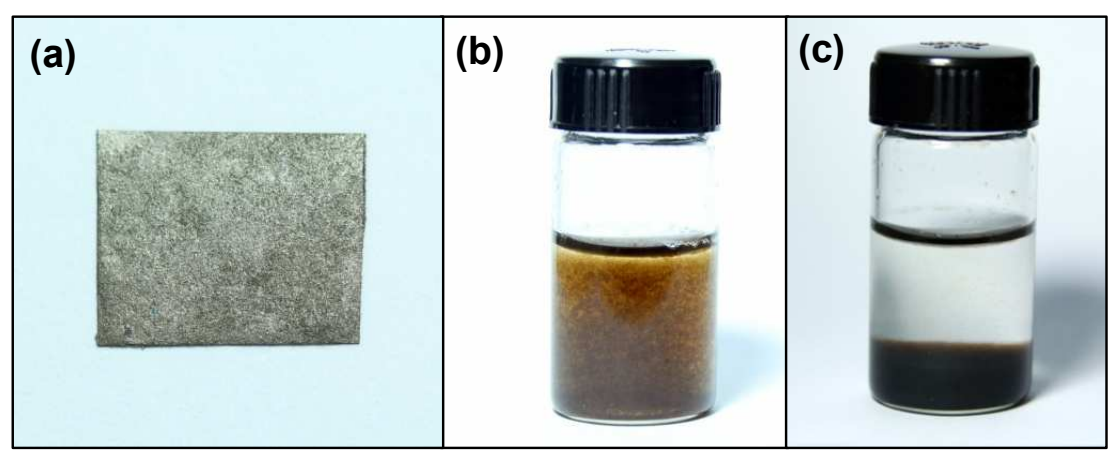

Figure S5. (a) Digital picture of GOA after being compressed at $140 \mathrm{MPa}$. (b) Digital picture of compressed GOA immediately after being stirred in water for $24 \mathrm{~h}$. (c) Digital picture of the dispersion in (b) after sedimentation of 3 min. 

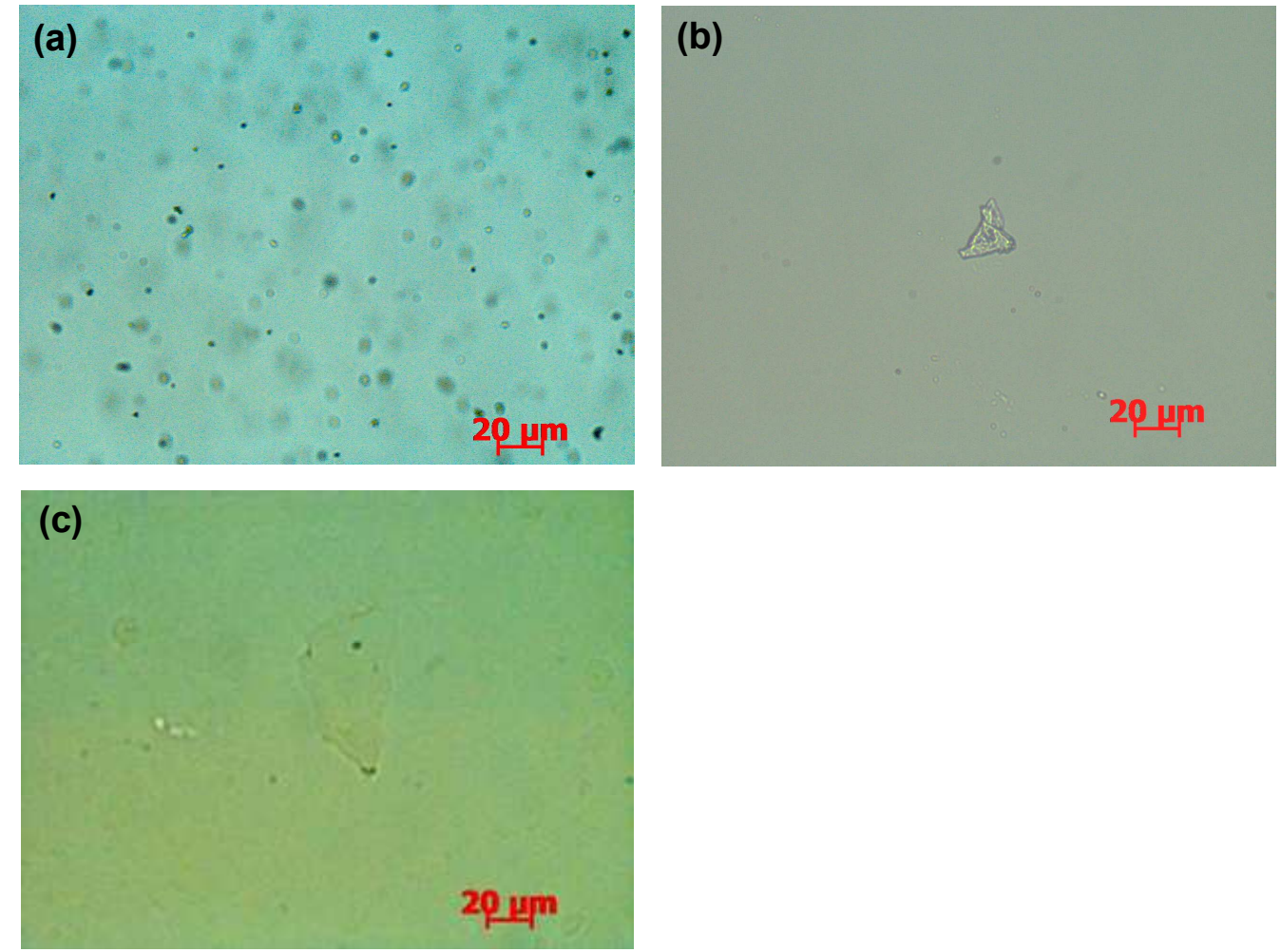

Figure S6. High-solution optical images of fGO after shaked in $\mathrm{H}_{2} \mathrm{O}$ forl min (a),15 $\min (\mathbf{b}), 1 \mathrm{~h}(\mathbf{c})$. 

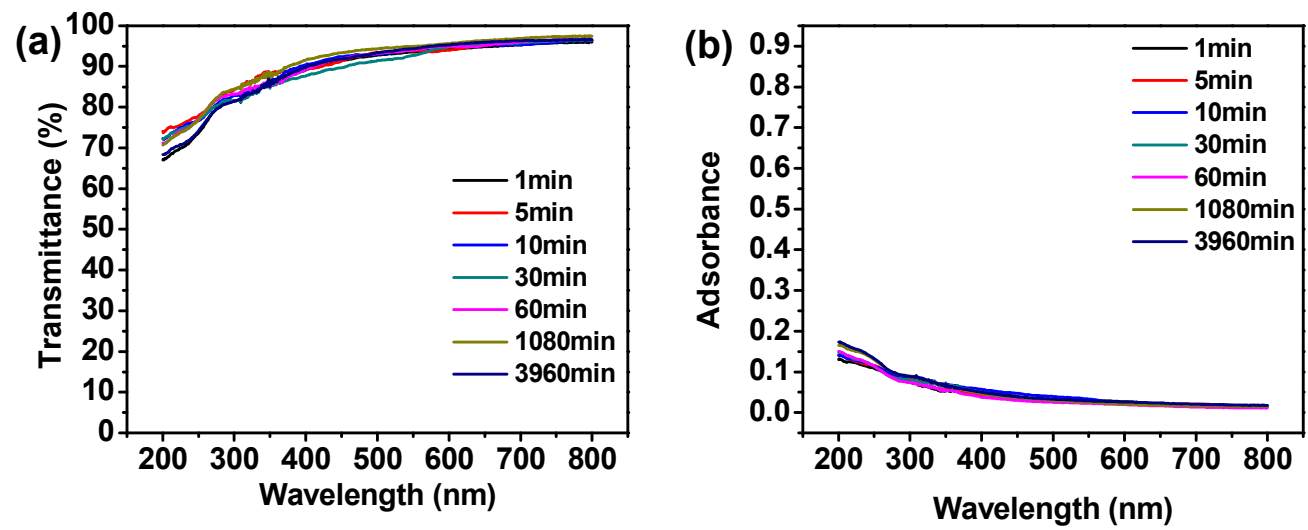

Figure S7. UV-Vis spectra of GOA dispersed in water at $0.02 \mathrm{mg} / \mathrm{g}$ after stirring at $500 \mathrm{rpm}$ for different time. 

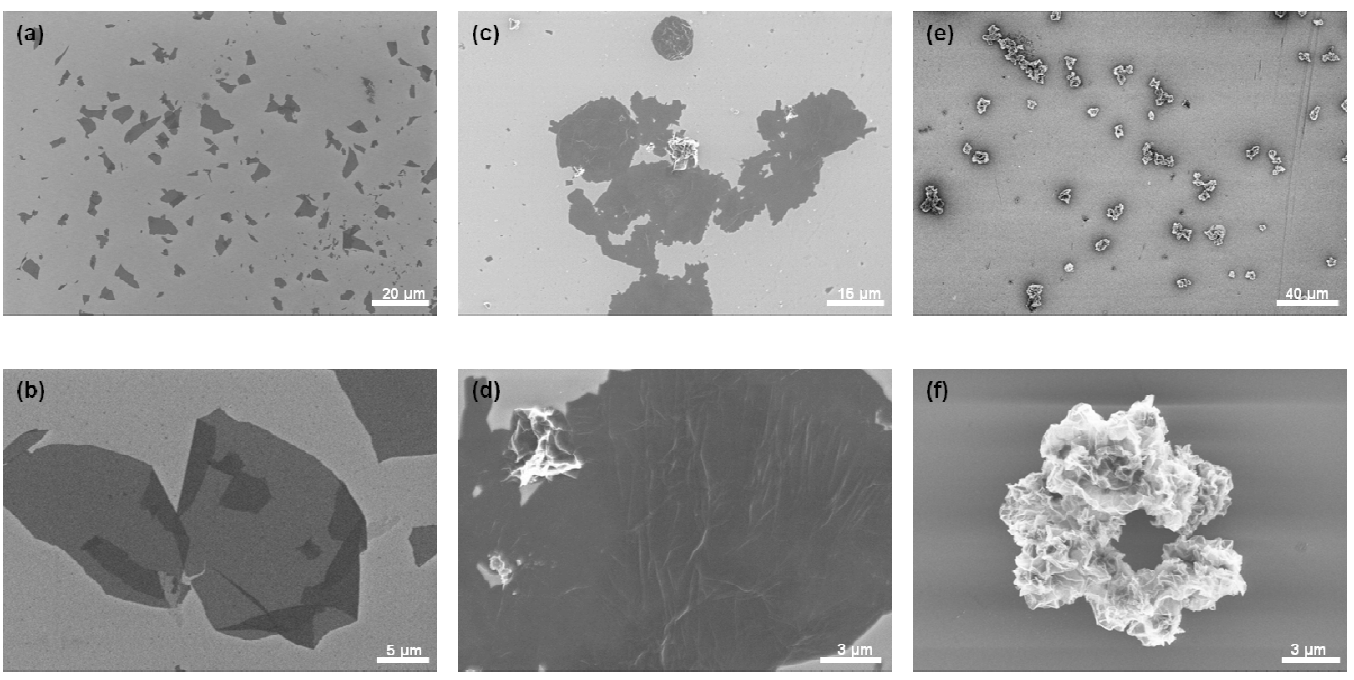

Figure S8. SEM images of fGO after stirring at $500 \mathrm{rpm}$ for $24 \mathrm{~h}$ in DMF (a, b), ethanol (c, d), acetone (e, f). 

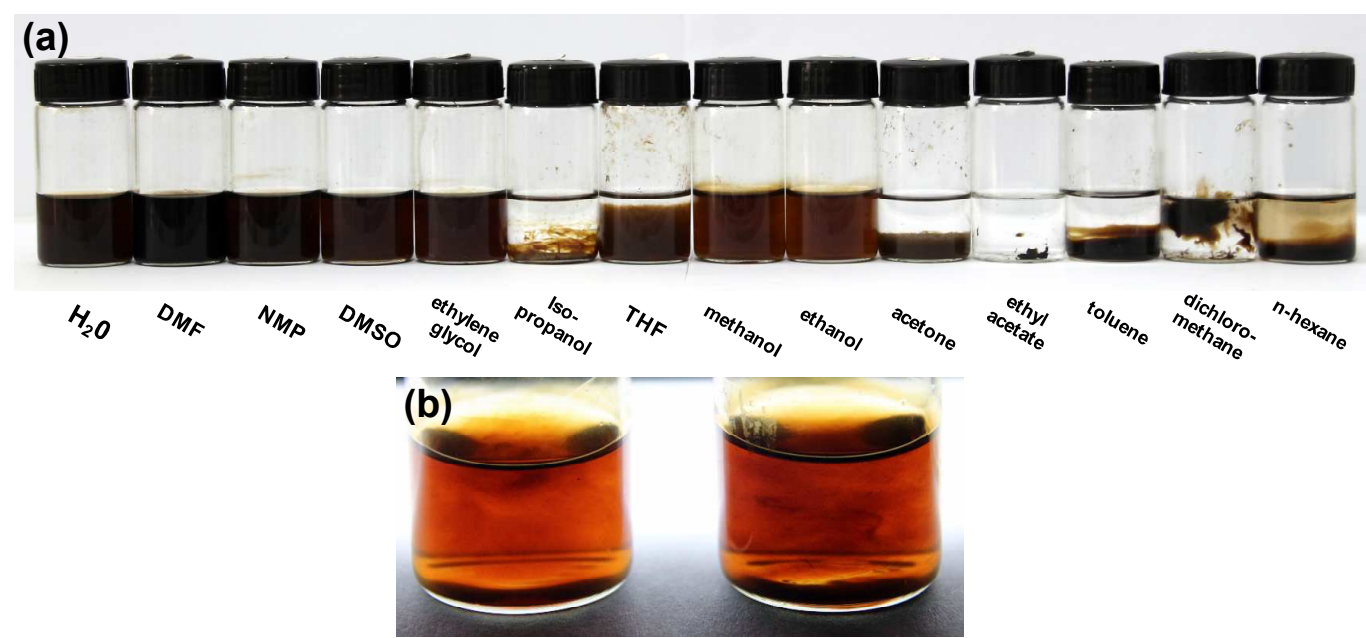

Figure S9. (a) Digital picture of GO suspensions in 14 different solvents after sedimentation of 7 days. (b) Close observation of GO dispersions in methanol (left) and ethanol (right). 
(a)

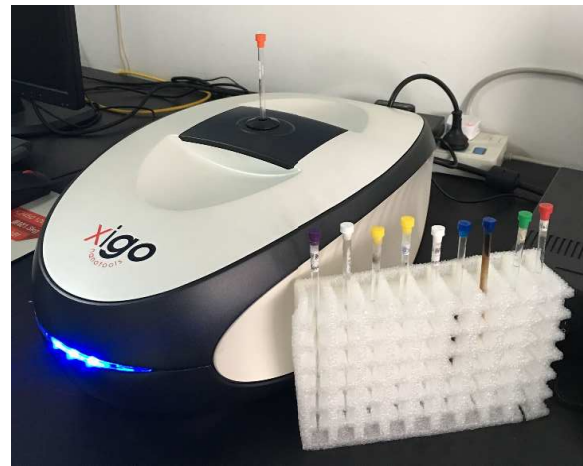

(b)

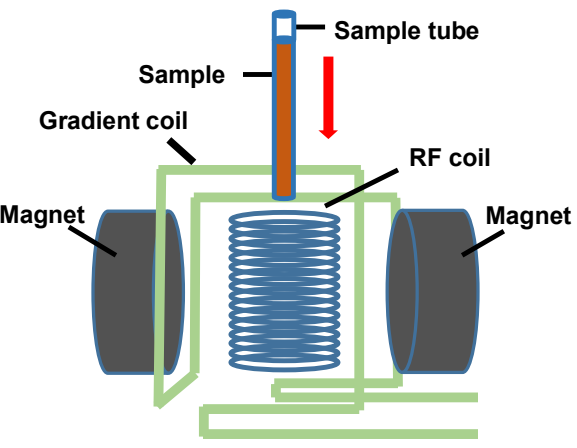

Figure S10. (a) Digital picture of a Acorn Area ${ }^{\mathrm{TM}}$. (b) Schematic illustrating the test principle of NMR solvent relaxation. 